\title{
Gastrointestinal endoscopy during the coronavirus pandemic in the New York area: results from a multi-institutional survey
}

다(1)우우

\author{
Authors \\ Institutions \\ 1 NYP-Weill Cornell Medicine, New York, New York, \\ United States \\ 2 Stony Brook University Hospital, Stony Brook, NY \\ 3 NYP-Columbia University Irving Medical Center, New \\ York, United States \\ 4 NYU Langone Health, New York, New York, United States \\ 5 Northwell Health, Manhasset, NY \\ 6 Mount Sinai Hospital, New York, New York, United States \\ 7 Memorial Sloan Kettering Hospital, New York, New York, \\ United States
}

Srihari Mahadev', Olga C. Aroniadis², Luis H. Barraza3, Emil Agarunov³, Michael S. Smith6, Adam J. Goodman", Petros C. Benias ${ }^{5}$, Jonathan M. Buscaglia ${ }^{2}$, Seth A. Gross ${ }^{4}$, Franklin Kasmin ${ }^{6}$, Jonathan Cohen ${ }^{4}$, David L. Carr-Locke ${ }^{1}$, David Greenwald ${ }^{6}$, Robin Mendelsohn ${ }^{7}$, Amrita Sethi ${ }^{3}$, Tamas A. Gonda ${ }^{3}$, (on behalf of NYSGE research committee)

submitted 25.6.2020

accepted after revision 20.8.2020

Bibliography

Endoscopy International Open 2020; 08: E1865-E1871

DOI 10.1055/a-1264-7599

ISSN 2364-3722

(c) 2020. The Author(s).

This is an open access article published by Thieme under the terms of the Creative Commons Attribution-NonDerivative-NonCommercial License, permitting copying and reproduction so long as the original work is given appropriate credit. Contents may not be used for commecial purposes, or adapted, remixed, transformed or built upon. (https://creativecommons.org/licenses/by-nc-nd/4.0/)

Corresponding author

Tamas Gonda, MD, Division of Gastroenterology and Hepatology, New York University, New York, United States tamas.gonda@nyulangone.org

Supplementary material is available under https://doi.org/10.1055/a-1264-7599

\section{ABSTRACT}

Background and study aims The coronavirus disease 2019 (COVID-19), and measures taken to mitigate its impact, have profoundly affected the clinical care of gastroen- terology patients and the work of endoscopy units. We aimed to describe the clinical care delivered by gastroenterologists and the type of procedures performed during the early to peak period of the pandemic.

Methods Endoscopy leaders in the New York region were invited to participate in an electronic survey describing operations and clinical service. Surveys were distributed on April 7, 2020 and responses were collected over the following week. A follow-up survey was distributed on April 20, 2020. Participants were asked to report procedure volumes and patient characteristics, as well protocols for staffing and testing for COVID-19.

Results Eleven large academic endoscopy units in the New York City region responded to the survey, representing every major hospital system. COVID patients occupied an average of $54.5 \%(18-84 \%)$ of hospital beds at the time of survey completion, with $14.5 \%$ ( $2 \%-23 \%$ ) of COVID patients requiring intensive care. Endoscopy procedure volume and the number of physicians performing procedures declined by $90 \%$ (66\%-98\%) and $84.5 \%$ (50\%-97\%) respectively following introduction of restricted practice. During this period the most common procedures were EGDs (7.9/unit/ week; $88 \%$ for bleeding; the remainder for foreign body and feeding tube placement); ERCPs (5/unit/week; for cholangitis in $67 \%$ and obstructive jaundice in $20 \%$ ); Colonoscopies (4/unit/week for bleeding in $77 \%$ or colitis in $23 \%$ ) and least common were EUS (3/unit/week for tumor biopsies). Of the sites, $44 \%$ performed pre-procedure COVID testing and the proportion of COVID-positive patients undergoing procedures was $4.6 \%$ in the first 2 weeks and up to $19.6 \%$ in the subsequent 2 weeks. The majority of COVID-positive patients undergoing procedures underwent EGD (30.6\% COVID +) and ERCP (10.2\% COVID +).

Conclusions COVID-19 has profoundly impacted the operation of endoscopy units in the New York region. Our data show the impact of a restricted emergency practice on endoscopy volumes and the proportion of expected COVID positive cases during the peak time of the pandemic. 


\section{Introduction}

Since the first case of the novel 2019 coronavirus disease (COVID-19) was identified in Wuhan, China on November 17, 2019, the disease has spread across the globe and infected over 4.9 million individuals, resulting in over 328,000 deaths [1]. These figures likely underestimate of the true burden of COVID-19 due to uncounted deaths outside of medical settings as well as the high prevalence of asymptomatic or minimally symptomatic carriers, which facilitates rapid transmission in densely populated areas [2]. Currently, the United States has the highest caseload worldwide, and since identifying its first case on March 1, 2020, New York City has emerged as the world epicenter of the pandemic [3]. COVID-19 has profoundly affected the care of patients with gastrointestinal diseases, both through measures implemented to mitigate the spread of the virus, and through diversion of human and material resources from gastroenterology services to areas in greater need during this crisis.

Early in the course of the pandemic, endoscopy societies around the world recommended restricting the practices of endoscopy units to protect patients, frontline healthcare workers (HCWs), and to conserve personal protective equipment (PPE). In the United States the major professional gastroenterology societies issued a joint statement recommending that endoscopy units strongly consider rescheduling elective, nonurgent endoscopic procedures [4]. These recommendations aligned with guidance from the US surgeon general calling for cancellation of elective surgery. Additional recommendations were issued regarding the pre-screening of all patients undergoing endoscopy with history of high-risk exposure, including travel to areas with a high prevalence of COVID-19. For endoscopy units located in New York City, these multi-society guidelines implied that essentially all patients should be considered high risk for COVID-19 exposure.

In light of evidence that SARS-CoV-2, the virus that causes COVID-19, is present in the GI tract and potentially transmissible through secretions $[5,6]$, the use of enhanced contact and droplet splash precautions as well as the regular use of aerosol filtering face-piece respirators has been recommended $[7,8]$. It is believed that both upper and lower endoscopy have the potential for aerosol generation due to the insufflation and aspiration of digestive secretions required during routine procedures. Repici et al reported their experience from Italy, once the epicenter of the pandemic, and proposed universal use of respirator masks, a hairnet, goggles/face shield, two pairs of gloves, and a gown for high risk patients (or patients in highprevalence areas). Due to shortages of PPE, particularly of N95 respirators, the extent to which these recommendations are being followed in practice remains unknown. In New York, HCWs have reported significant difficulty securing masks and other PPE, and it is unknown to what extent these issues have affected endoscopy staff and units in this region [9].

Despite the cancellation of elective procedures, there remains a steady influx of patients requiring urgent inpatient or outpatient endoscopy. Our aim was to describe the approach taken by endoscopy units operating during the COVID-19 pan- demic, both in terms of characterizing the type of procedures performed during this period, as well as the operational measures implemented including endoscopy suite staffing, PPE utilization, and pre-procedure COVID-19 testing.

\section{Methods}

\section{Survey design}

A web-based survey was distributed via the New York Society for Gastrointestinal Endoscopy (NYSGE) list-serve. Each individual NYSGE member had the opportunity to designate one member of their endoscopy faculty to contribute institutional based data. In addition, direct emails were sent to all endoscopy directors and division chiefs in the NY region. As our goal was to provide time-sensitive information, distribution was limited to this area given the early clustering of cases.

The surveys were exempt from Institutional Review Board approvals as they were entirely anonymous and did not contain identifiable information. However, each respondent was asked to confirm this at their respective institutions. Subsequently each respondent was asked to enter the data in a REDCap database that was housed at Columbia University Irving Medical Center.

An institutional identifier was used for the sole purpose of recognizing multiple entries from the same institution. As several hospital systems comprise multiple larger hospitals, data entered separately for each hospital was treated as distinct.

Given the daily fluidity of the numbers, we asked respondents to average the values, when appropriate, based on the time period of the questionnaire.

The first questionnaire was distributed on 3/30/2020 and data was collected until April 12, 2020 (representing the first 2 weeks of the pandemic). This survey is found in the Supplementary Methods, Appendix. An updated survey targeting the changes in response to the pandemic at its peak in this region was distributed on April 20, 2020 and data were collected through May 2, 2020.

\section{Survey data reporting and analysis}

We collected data on the number of attending and trainee physicians, nurses and other staff members in the endoscopy unit prior to and during the pandemic.

Baseline data on the weekly number and type of endoscopies performed was collected. Subsequently, data regarding procedures performed at the two time points in the endoscopy unit and the ICU was collected. Patients with confirmed COVID infection at the time of endoscopy were also assessed. Continuous variables such as procedure volume were summarized using means and interquartile ranges (IQR) and were compared between different time points using a paired sample $t$-test. Categorical variables such as procedural indications were reported as the percentage of institutions performing a specific procedure for that indication.

In addition, data were collected regarding staff exposure, guidance on procedural prioritization and PPE use. 


\section{Results}

\section{Characteristics of surveyed institutions}

Eleven large primarily academic endoscopy units in the New York City region responded. Data were collected from a total of seven hospital systems; four systems contributed data from one hospital endoscopy unit each (Montefiore Medical Center, Mount Sinai Hospital, Memorial Sloan Kettering, Northwell Health) while the remaining three systems contributed data from multiple units (New York University: Tisch Hospital, Bellevue Hospital, and Ambulatory Care Center; New York Presbyterian: Weill Cornell Medical Center and Columbia University Irving Medical Center; State University of New York: Downstate and Stony Brook Medical Centers). We estimated the number of COVID + inpatients and critically ill (ICU) patients based on an average at two time points and based on publicly available reporting from each institution to their faculty and staff. The data are summarized in > Table 1.

\section{Staffing}

Endoscopy physician staffing levels were cut markedly during the pandemic. These data are summarized in > Table 2 . The number of attendings performing endoscopy during the first 3 weeks of the pandemic decreased from 19.2 to 4.4 per unit, representing a $76 \%$ reduction from baseline staffing levels ( $P=$ $0.014)$. The number of fellows performing endoscopy decreased from 13.4 to 0.6 per unit, or a decrease of $96 \%(P<$ $0.001)$. At the time of the initial survey, $80 \%$ of attendings were not performing procedures with trainees. At the time of the second survey, $60 \%$ of attendings were not performing procedures with trainees.

\section{Overall endoscopy procedures volumes}

There was a dramatic and significant reduction in total volume of all procedures between the first survey and baseline data ( $>$ Fig. 1a), with total procedure volumes declining from a mean of 192 cases per week per unit to a mean 19 per week $(P<0.001)$. There was a modest, non-significant increase in total number of cases between the first and second questionnaire $(P=0.42)$. Of note, there was a significant increase in the number of COVID-positive patients undergoing endoscopy between the first and second time points, 0.9 vs 4.8 patients per week per unit respectively ( $P<0.001)$, ( $\mathbf{F i g . 1 b}$ ).

\section{Procedure indications}

The most common indication for both upper and lower endoscopy, which were performed at all institutions during the pandemic, was bleeding. All sites reported performing EGD and colonoscopy for this indication at the second time point. In addition, the majority of centers reported performing ERCP for cholangitis and obstructive jaundice during both time periods. In addition, at the time of the second survey, foreign body extraction, feeding tube placement and upper endoscopic stent placement was performed in $>60 \%$ of the centers. Other common gastrointestinal procedures were performed at less than half of the centers surveyed ( $\triangleright$ Fig. 2 ).

- Table 1 Institutional burden of COVID infections.

\begin{tabular}{|l|l|l|l|l|}
\hline & Patient census N (n)* & Percent of total beds & $\begin{array}{l}\text { Mean census per } \\
\text { institution }\end{array}$ & $\begin{array}{l}\text { Range per institu- } \\
\text { tion/IQR }\end{array}$ \\
\hline Total hospital beds & & & 937.6 & $330-2650 / 599$ \\
\hline COVID + inpatients (weeks 1-2) & $9376(10)$ & $56.5 \%$ & 568 & $92-2162 / 172$ \\
\hline COVID ICU patients (weeks 1-2) & $1365(9)$ & $15.1 \%$ & 151.7 & $23-613 / 133.5$ \\
\hline COVID + inpatients (weeks 3-5) & $3139(7)$ & $57.6 \%$ & 448.4 & $107-1538 / 234$ \\
\hline COVID ICU patients (weeks 3-5) & $1121(7)$ & $20.6 \%$ & 160.1 & $35-571 / 134$ \\
\hline$*$ N, total number of patients and staff; n, number of institutions able to provide data. & & \\
\hline
\end{tabular}

- Table 2 Staffing levels in gastrointestinal endoscopy units during the COVID pandemic.

\begin{tabular}{|l|l|l|l|}
\hline & $\begin{array}{l}\text { Total staff across all } \\
\text { institutions N (n) }\end{array}$ & $\begin{array}{l}\text { Mean staff per } \\
\text { institution }\end{array}$ & $\begin{array}{l}\text { Range per institu- } \\
\text { tion/IQR }\end{array}$ \\
\hline Gastrointestinal attendings pre-COVID & $173(9)$ & 19.2 & $8-50 / 21.5$ \\
\hline Gastrointestinal fellows pre-COVID & $134(10)$ & 13.4 & $8-18 / 7$ \\
\hline Gastrointestinal attendings performing procedures (weeks 1-2) & $42(10)$ & 4.2 & $2-10 / 4$ \\
\hline Gastrointestinal fellows post-COVID (weeks 1 - 2) & $6(10)$ & 0.6 & $0-3 / 1.25$ \\
\hline${ }^{*}$ N, total number of patients and staff; n, number of institutions able to provide data. & \\
\hline
\end{tabular}



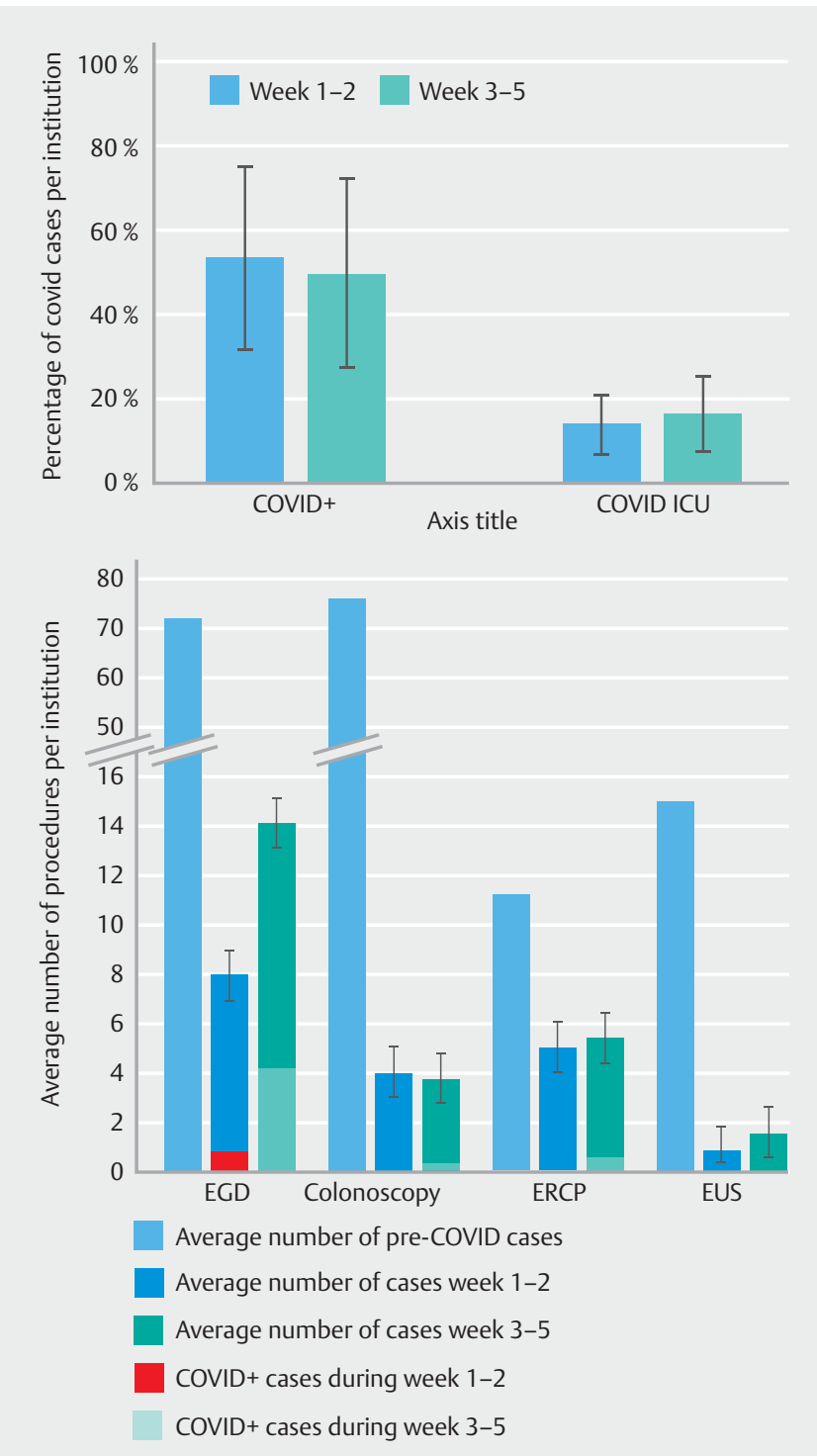

- Fig. 1 Proportion of COVID + cases and average number of endoscopic procedures performed at surveyed institutions. a Percentage of COVID + cases and COVID + patients in ICUs among all hospitalized patients in institutions surveyed. b Average number of endoscopies performed per week. The percentage of COVID + cases is indicated.

\section{COVID testing and availability of personal protective equipment}

Pre-procedure COVID-19 testing increased between the first and second surveys, with $44 \%$ and $60 \%$ of centers offering preprocedure testing for both inpatient and outpatient procedures at the time of the second survey. Most endoscopy units, $90 \%$ in our study, reported the use of a structured protocol to identify appropriate patients for endoscopy. None of the participating institutions reported a shortage of PPE ( $\triangleright$ Fig. 3 ).

\section{Discussion}

In this survey of endoscopy leaders from 11 large academic centers in the New York region, we describe the operations of endoscopy units from every major hospital system in the epicenter of the COVID-19 pandemic during the early to peak pandemic period. Our study demonstrates that while some aspects of the response to the pandemic have been consistent across centers, there is significant variation in key areas, including pre-procedure testing for COVID-19 among patients who require endoscopy. A consistent finding in this study is that endoscopy volumes declined markedly from baseline in accordance with directives from government and professional societies to postpone and cancel all non-urgent, elective cases. By surveying unit leaders on the types of procedures performed during the pandemic, we were able to characterize the indications that were considered sufficiently urgent to warrant intervention during the crisis, which may be instructive to other gastroenterology departments who find themselves in an earlier stage in the natural history of the pandemic.

Overall procedure volumes experienced a marked decline compared to baseline across all procedure types due to cancellation of elective cases. These findings highlight the fact that a large proportion of the work done in endoscopy units, even in large academic medical centers, consists of screening, surveillance, and other elective and semi-elective indications that can be safely deferred during a crisis to conserve resources. These are profound alterations in practice that are essentially without precedent in the modern era of gastrointestinal endoscopy. Our findings with regard to procedure volume - while extreme - are consistent with reports from other parts of the world, including China [10], Italy [11], as well as a multicenter survey from the United States and Canada, who describe reductions in overall procedure volume of $75 \%$ to $99 \%$, with most centers operating at $\leq 10 \%$ of their normal endoscopy volume [12]. These findings are also consistent with a survey of 69 individual gastroenterologists (as opposed to endoscopy unit leaders) from the New York area who report individual procedure volumes falling to zero cases in the preceding 7 days for $62 \%$ of respondents, and weekly endoscopy volumes declining by $57 \%$ to $96 \%$ across all procedure types [13].

Although there has been general consensus among professional societies that elective cases should be postponed, it remains unclear how restrictive the threshold for urgent and emergent cases should be, and which particular indications should continue to be performed. Our survey found several indications that were consistently considered urgent enough to warrant proceeding during the pandemic across all centers: primary inpatient general endoscopic procedures for an indication of gastrointestinal bleeding, gastrostomy tube placement, and biliary endoscopy for the management of obstructive jaundice and cholangitis. These findings are consistent with a study that surveyed gastroenterologists via social media on indications for endoscopy during the COVID-19 pandemic, where significant variability was found regarding recommendations on timing of endoscopy for the majority of indications, however relative consensus was present for performing endoscopy for suspect- 


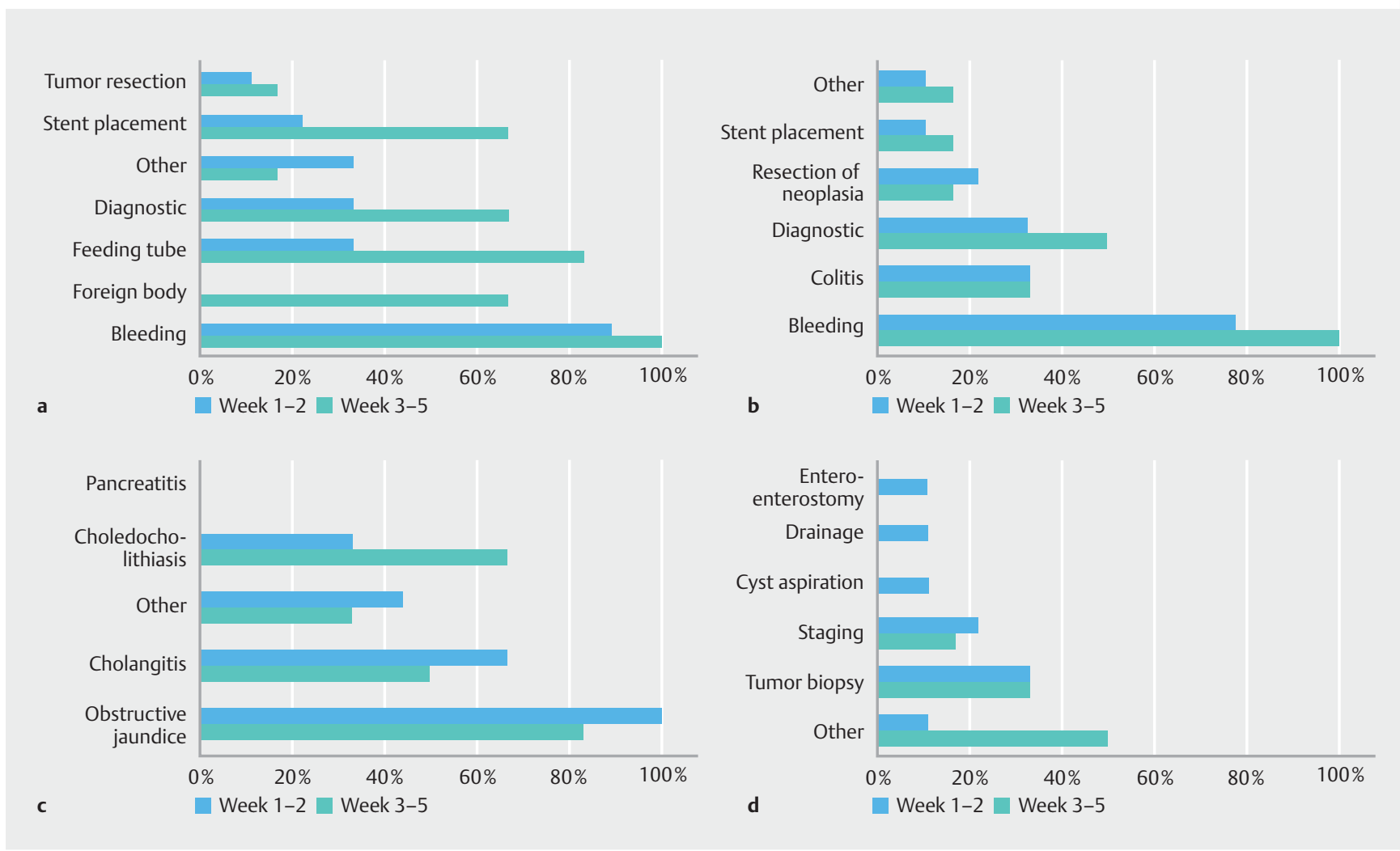

- Fig. 2 Endoscopic procedures during the COVID Pandemic. a Percentage of EGD indications performed at each institution. $\mathbf{b}$ Percentage of colonoscopy indications performed at each institution. $\mathbf{c}$ Percentage of ERCP indications performed at each institution. $\mathbf{d}$ Percentage of EUS indications performed at each institution.

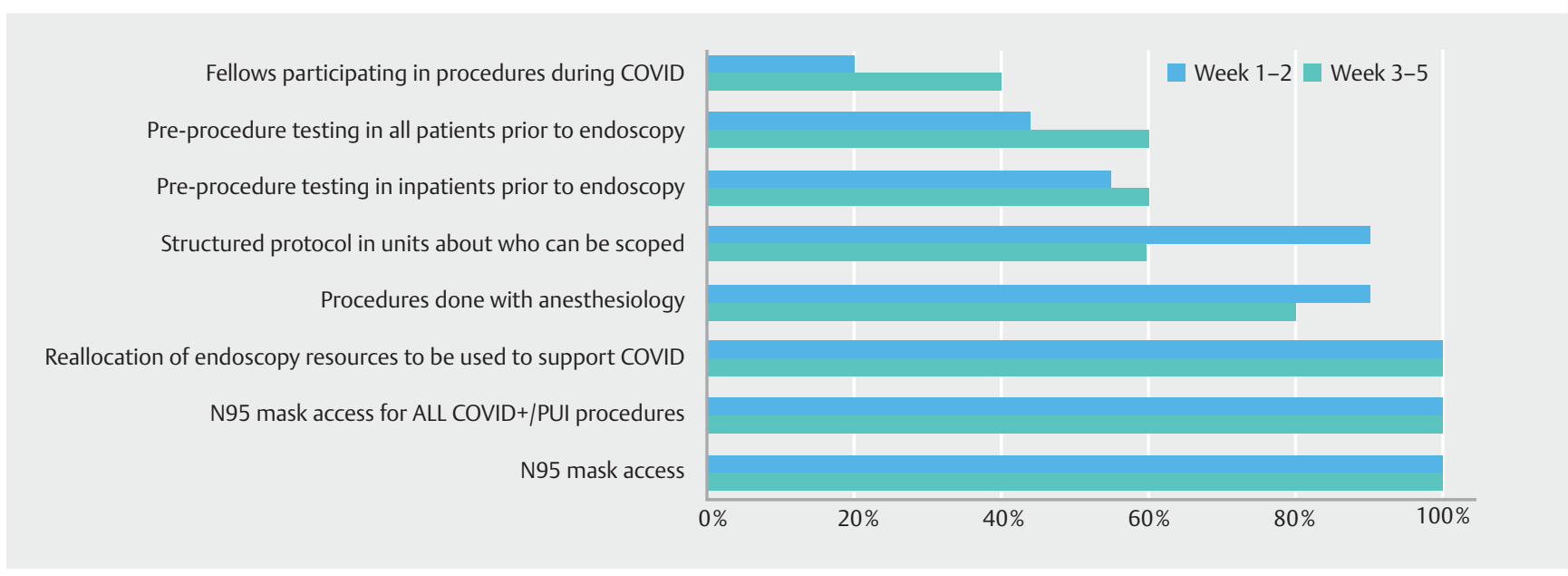

- Fig. 3 COVID testing and Personal protective equipment (PPE) use at each institution. a Percentage of institutions adopting practices and PPE use during the pandemic.

ed upper gastrointestinal bleeding, symptomatic choledocholithiasis or cholangitis [14]. In this survey, the majority of respondents thought only patients with hemodynamic instability due to bleeding should get inpatient colonoscopy. Our findings reveal colonoscopy was performed for the indication of gastrointestinal bleeding at roughly half the rate of upper endoscopy for this indication, suggesting that clinicians in New York continue to evaluate hematochezia with both endoscopy and colo- noscopy at reasonable frequency. Another study attempted to reach a consensus amongst 14 invited expert endoscopists on the degree of urgency of 41 common indications for advanced endoscopy [15]. High rates of consensus were obtained for proceeding within 1 week in patients with indications of jaundice with or without cholangitis, dysphagia, gastric outlet obstruction, or bowel obstruction requiring intervention, as well as 
endoscopic drainage of symptomatic fluid collections. Gastrointestinal bleeding was not evaluated in this study.

Significant variability was found in this survey between centers regarding pre-endoscopy COVID-19 testing procedures. This variability may reflect the initial scarcity of testing resources during the early phase of the pandemic in New York. We found a slight increase in testing rates between the first and second survey, which coincides with increased testing capability. Of note, testing was not associated with an increase in procedure volumes, likely because testing was instituted prior to resumption of elective procedures. There is significant evidence that COVID-19 is frequently associated with gastrointestinal symptoms, in particular diarrhea-with one-third of patients reporting digestive symptoms in a large cohort from New York City [16]. This raises the possibility that COVID-19 may represent an etiology of gastrointestinal symptoms that may prompt investigation, and possibly endoscopy, in patients who present with extra-pulmonary COVID-19 symptoms, especially as units begin to resume semi-elective and elective procedures. These facts support the practice of universal testing for patients undergoing endoscopy, assuming the risk of transmission is significant and that deferring endoscopy until such time as the patient is no longer polymerase chain reaction-positive is feasible. The true risk of exposure to gastroenterologists and endoscopy staff on COVID-19 positive patients is unknown. One study of patients and HCWs practicing at 41 endoscopy units in Italy during the pandemic found that $4.3 \%$ (including 23 physicians) contracted COVID-19, of whom $14.3 \%$ required hospitalization for interstitial pneumonia [17]. The majority of centers in this study (71\%) reported no cases of infection amongst their endoscopy teams. While the authors interpret these findings to suggest a low risk of COVID-19 infection from performing endoscopy, we argue that this rate of infection was unacceptably considering the marked reduction in endoscopy volume and the $10 \%$ baseline infection rate among HCWs in Italy at the time of that survey. As we consider how to reopen endoscopy units and resume the care of less urgent but nevertheless important indications for endoscopy, testing will undoubtedly be a central part of the strategy to minimize the risk of infection of staff and COVID-19 negative patients who are present in the facility, and we suspect rates of testing will increase over time.

The centers who participated in this study were overwhelmingly large academic institutions, and this provided information regarding the involvement of fellows in procedures during the pandemic. The majority of centers did not allow fellows to participate in procedures during the pandemic, however these restrictions appear to have eased somewhat between the two survey timepoints. These findings are consistent with recommendations from endoscopy societies to limit trainee involvement in order to minimize procedure duration, reduce the number of individuals exposed in the procedure room, and to conserve PPE [18]. Given the uncertain timeframe of the pandemic and restrictions required to mitigate its spread, the impact on skills of fellows and competency acquisition may be considerable. These restrictions are particularly important for advanced endoscopy fellows whose training time is finite and who have little opportunity to recoup training opportunities, even as endoscopy units begin to open at a fraction of their previous capacity. This secondary effect warrants further study.

There are several implications of our study for what needs to come next: reopening and resuming normal gastrointestinal endoscopy operations. The American Society for Gastrointestinal Endoscopy (ASGE) has made several key recommendations, including pre-procedure screening of all patients with a dedicated COVID-19 screening questionnaire, however they stopped short of recommending universal viral testing for all patients prior to endoscopy. Our study demonstrates that a significant proportion of centers are already testing for COVID-19 in all patients undergoing endoscopy, and as availability of testing materials increases, we anticipate a move toward universal preprocedure testing. The ASGE also recommends universal N95 respirator use for all members of the endoscopy team. Our study indicates that these measures are currently in place in all the surveyed centers and supports their universal implementation as feasible. Given the significant discrepancies we have demonstrated between baseline and pandemic era procedure volumes across all procedure types, significant challenges will no doubt arise with the scaling up of operations to fulfill the backlog of deferred cases and return to volumes approaching baseline.

Our study has several strengths. Most importantly, all major academic hospital systems in New York City were included, hence the survey is able to present a comprehensive picture of city-wide endoscopy activities in the epicenter of the COVID-19 pandemic during the beginning and peak of the pandemic. The cooperative involvement of multiple institutions in the survey reduces the likelihood that procedure volume was diverted from one institution to another, and not captured in the survey. There are several limitations however. The survey targeted large academic hospitals, and did not include community hospitals, ambulatory surgery centers, or office-based procedures. During the beginning and peak of the pandemic, these centers were likely to have been shut down, hence it is unlikely they would account for a significant proportion of endoscopy taking place in the New York City area, however, as centers reopen, survey results that exclude these centers will likely be biased. The survey requested aggregate, de-identified data and hence specific demographic and clinical information for each patient was not available.

\section{Conclusion}

In summary, our survey demonstrates the profound impact COVID-19 has had on the care of patients with gastrointestinal diseases, with marked reductions in endoscopy volumes. As the peak of the pandemic passes and we plan ahead to resuming elective procedures, we expect a large backlog of cases that will present challenges given the novel additional burdens of pre-screening, testing, social distancing, and personal protective equipment that now accompany the operations of endoscopy units. 


\section{Competing interests}

The authors declare that they have no conflict of interest.

References

[1] World Health Organization. COVID-19 Situation Report. Situation Report -114 https://www.who.int/docs/default-source/coronaviruse/situation-reports/20200513-covid-19-sitrep-114.pdf? sfvrsn=17ebbbe_4 Published May 13, 2020. Accessed May 14, 2020.

[2] Li R, Pei S, Chen B et al. Substantial undocumented infection facilitates the rapid dissemination of novel coronavirus (SARS-CoV-2). Science 2020; 368: 489-493

[3] Wadhera RK, Wadhera P, Gaba P et al. Variation in COVID-19 Hospitalizations and Deaths Across New York City Boroughs. JAMA 2020; 323: 2192-2195

[4] Joint GI society message: COVID-19 clinical insights for our community of gastroenterologists and gastroenterology care providers. https://www.asge.org/home/joint-gi-society-message-covid-19 Accessed May 14, 2020

[5] Sethi A, Swaminath A, Latorre M et al. Donning a new approach to the practice of gastroenterology: perspectives from the COVID-19 pandemic epicenter. Clin Gastroenterol Hepatol Off Clin Pract J Am Gastroenterol Assoc 2020; 18: 1673-1681

[6] Gu J, Han B, Wang J. COVID-19: Gastrointestinal manifestations and potential fecal-oral transmission. Gastroenterology 2020; 158: 1518-1519

[7] Xiao F, Tang M, Zheng $X$ et al. Evidence for gastrointestinal infection of SARS-CoV-2. Gastroenterology 2020; 158: 1831-1833.e3

[8] Carr-Locke DL, Soetikno R, Sharaiha RZ. Whatever happened to universal/standard precautions? Gastrointest Endosc 2020; 92: 399-400
[9] Repici A, Maselli R, Colombo M et al. Coronavirus (COVID-19) outbreak: what the department of endoscopy should know. Gastrointest Endosc 2020; 92: 192-197

[10] Kamerow D. Covid-19: the crisis of personal protective equipment in the US. BMJ 2020; 369: m1367

[11] Repici A, Pace F, Gabbiadini R et al. Endoscopy units and the COVID-19 outbreak: a multi-center experience from Italy. Gastroenterology 2020; 159: 363-366.e3

[12] Forbes N, Smith ZL, Spitzer RL et al. Changes in gastroenterology and endoscopy practices in response to the COVID-19 Pandemic: results from a North American survey. Gastroenterology 2020; 159: 772774.e13

[13] Mahadev S, Aroniadis OS, Barraza L et al. Impact of the COVID-19 pandemic on endoscopy practice: results of a cross-sectional survey from the New York metropolitan area. Gastrointest Endosc 2020; 92: 788-789

[14] Bilal M, Simons M, Rahman AU et al. What constitutes urgent endoscopy? A social media snapshot of gastroenterologists' views during the COVID-19 pandemic Endosc Int Open 2020; 8: E693-E698

[15] Sawhney MS, Bilal M, Pohl H. Triaging advanced Gl endoscopy procedures during the COVID-19 Pandemic: consensus recommendations using the Delphi method. Gastrointest Endoscop 2020; 92: 535-542

[16] Hajifathalian K, Krisko T, Mehta A et al. Gastrointestinal and Hepatic Manifestations of 2019 Novel Coronavirus Disease in a Large Cohort of Infected Patients From New York: Clinical Implications. Gastroenterology 2020; 159: 1137-1140.e2

[17] Repici A, Aragona G, Cengia G et al. Low risk of covid-19 transmission in Gl endoscopy. Gut 2020: doi:10.1136/gutjnl-2020-321341

[18] Hennessy B, Vicari J, Bernstein B et al. Guidance for resuming GI endoscopy and practice operations after the COVID-19 pandemic. Gastrointest Endosc 2020; 92: 743-747.e1 